\title{
An Eco-friendly Alternative to Polyethylene Microbeads in Personal Healthcare Products
}

\author{
Siksha G Shankla, Manisha Agrawal ,Megha Kumari, Samyuktha, Amantrika Gupta \\ Communicating author: Dr. Danie Kingsley J* \\ Asst prof sr, SBST, VIT Vellore 632014
}

\begin{abstract}
Recently a huge chunk of the population has shifted towards shower gels. The biggest reason might be the fact that shower gels can provide a better care for the skin. They were made with the motive of providing gentle cleansing and moisturizing qualities contrary to that of a regular soap, so that the people can enjoy a better skin care experience. Shower gels generally use chemicals especially sodium lauryl sulfate and betaines. These are believed to cause skin irritation and may not be washed off properly especially in hard water. The recent shower gels have a combined scrubbing effect included along with the scented or flavored body wash. For the scrubbing activity microbeads are employed. These microbeads used as a scrubber are polymers, e.g. being polyethylene or nylon etc. We know how plastics are harmful to us and the environment. And this is a cause of major concern. And since it is small in size it makes it relatively dangerous. Microbeads, because of their small size, have a large surface area by volume which allows them to accumulate highly toxic materials which are toxic to the person and the environment. To overcome the menace of microbeads we propose an alternative. This alternative aims to use biodegradable waste products like pistachio shells and orange peels to substitute the scrubbing and cleansing activity of the microbeads. We have also designed a compact structure of shower gel dispenser with attached loofa so that there is no need of buying a separate loofa thus providing a cost effective solution. We aim to make this product easy to use and travel friendly. [1][2]
\end{abstract}

Keywords:- Microbeads, Marine Life, Micro Plastic, Biodegradable Alternatives.

\section{INTRODUCTION}

While the world is being taken over by heaps and heaps of plastic waste that can be seen in landfills as well as floating as debris in the water bodies, another imminent threat arises that is not visible to the naked eye. Many studies have reported that micro plastics have infiltrated the Earth's oceans and its aquatic ecosystem. They might not be visible but they cause a lot of harm to the ecosystem, by entering the food-chain or simply by being consumed by the aquatic organisms. Its small size makes it bioavailable to all trophic levels. Due to there being no efficient cleanup technology, scientists and environmentalists push towards the reduction of usage of micro plastics. [3]
One of the chief sources of micro plastics are plastic microbeads which is found in a lot of cosmetics like scrubs and exfoliating face washes. Microbeads are plastic beads, size ranging from $5 \mu \mathrm{m}$ to $1 \mathrm{~mm}$. They are chiefly made of synthetic polymers like polyethylene, polylactic acid (PLA), polypropylene, polystyrene, or polyethylene terephthalate. Microbeads finds its usage in a wide range of products, mostly as exfoliants and abrasives, which include shower gels, cleaning supplies, face cleansers and facial srubs. The natural alternatives to plastic microbeads are pumice, walnut shells and oatmeal [4]. Microbeads are designed to be a wash off product that contaminates the drain. Owing to its miniscule size they can escape the filtering process unscathed and find their way into the environment through the sewage-sludge from wastewater treatment plants (WWTPS). They have been reported to be one of the many types of plastic debris in marine habitats [5][6][7]. Micro plastics pose a huge ecological threat due to their ingestion by the aquatic wildlife. It has become a very commonly noted menace. Upon cutting open the guts of a wide variety of aquatic organisms like fishes, seabirds, whales and turtles to examine the cause of death, it has been discovered that the micro plastics resulted in the entanglement and blockage of the digestive system [8][9].

The prolonged presence of plastic in the water creates an additional indirect problem. Research shows that adsorption of persistent organic pollutants (POPs) onto the plastic occurs [8][10] [8][10]. POPs are distinguished by their persistence in the environment, long range movement, biomagnification and toxicity. The ads

The adsorption of POPs into plastic debris has been extensively reported, examples include polybrominated diphenyl ethers (PBDEs), polychlorinated biphenyls (PCBs), organochlorine pesticides, polyaromatic hydrocarbons (PAHs) and perfluorinated surfactants (PFCs), amongst others. Microplastics pollution has resulted in increasing public attention over the recent years as many cases of plastic ingestion by marine organisms have been pouring in. In light of this concerns over the potential bioaccumulation of toxic chemicals and its entrance into the food web has been expressed by scientists [11][12].

But the main problem in proving its [microplastics] presence in water bodies is the inability to capture it in sampling nets due to its small size [13]. However a study conducted in Honk Kong reported a case of microbeads with their size, shape, colour, polymer composition 
matching the microbeads found in personal care and composite products (PCCPs) [14].

In the light of stacking evidence that plastic microbeads are threatening the ecosystem, steps have to be taken to reduce this menace. So we have come up with an alternative microbead that is biodegradable, eco-friendly and harmless.

\section{MATERIALS AND METHODS}

\section{A. For shower gel}

\section{Materials:}

- Shea Butter - It acts as a very good moisturizing agent for skin, keeps it healthy and glowing.

- Oil \& Glycerin - it nourishes the skin and makes the skin soft

- Guar gum - It increases the viscosity and allows the solid particles (here scrub) to remain suspended in the liquid (shower gel).

- Castile soap - It is made from simple plant oils and it is considered safe for even the most sensitive skin types. The oils in castile soap penetrates through the pores with their antibacterial agents, and helps clean skin without drying it out and therefore it can also help oily, acne-prone skin.

- Warm water - acts as a solvent

- Orange and pistachios shell powder - used as biodegradable scrub particles.

- Rosemary essential oil - It is a natural anti-fungal, antibacterial and antiseptic oil, so it acts as an excellent preservative in personal care products and it is also a good skin tonic for any kind of skin conditions that are caused from excess bacteria or congested pores.

\section{$>$ Procedure:}

We are using shea butter as it is a moisturizer which helps to keep the skin healthy. First we melt the shea butter. Then we add the oil and glycerin and sprinkle the guar gum which increases the viscosity and allows the scrub particles to remain suspended. We then emulsify the mixture using an immersion blender. Then we add the rest of the ingredients namely castile soap for lathering, warm water which acts as a solvent, orange peel powder, pistachios shell powder for scrubbing property and the essential oils for good odor and antibacterial property . After blending the mixture we transfer the creamy gel to a squeeze bottle. [15][16]

\section{B. Scrub beads \\ > Procedure:}

Alginate is a natural polymer that satisfies our goal to create an eco-friendly product and it has also been verified to be safe for human use. To make the sodium alginate capsule we take $0.7 \mathrm{~g}$ of sodium Alginate in $40 \mathrm{ml}$ of water and prepare the solution $(1.75 \% \mathrm{w} / \mathrm{v})$. The orange peel powder was sprinkled on it and small droplets were pipetted into a $0.15 \mathrm{M}$ solution of $\mathrm{CaCl} 2(8.8 \mathrm{~g}$ in $40 \mathrm{ml})$.
Beads were then left undisturbed for $15 \mathrm{mins}$ filtered out and left to dry. The pistachios shells, essential oils and orange peel powder are added after performing sodium alginate encapsulation. This is to have an even distribution of the materials and to prevent them from settling down. [17][18][19].

\section{Uses of Orange and Pistachio in Shower Gel}

\section{- Orange [20]}

Orange is fruit enriched in natural oils and vitamins. It has been known for years as an ingredient in recipes, but recently they're being recognized for its beauty enhancing qualities. The benefits of orange for your skin are many, making it perfect to use in every aspect of our daily beauty regimes, because its natural properties will improve the look and feel of our skin. There is no reason to throw away the orange rind either, it's perfect for creating face masks and body scrubs, drawing out all the impurities leaving a gorgeous healthy glow.

There are many benefits of Orange for skin and here are the reasons why:

$\checkmark$ Natural oils in Oranges help to moisturize skin which provides softer healthier looking skin for longer.

$\checkmark$ Antioxidants found in Oranges helps to fight free radicals which may slow down the production of wrinkles, and stop premature aging.

$\checkmark$ Oranges have a high content of citric acid which aids in skin exfoliation and helps to dry out acne ultimately improving the overall look of your skin.

$\checkmark$ Orange peel has a higher content of Vitamin $\mathrm{C}$ than the orange itself, so grinded orange peel used as a body scrub in daily beauty regime gives healthy looking glow.

$\checkmark$ Vitamin C helps the body to form collagen and elastin which will help in skin looking younger.

$\checkmark$ Oranges could also prevent skin sagging.

- Orange Essential Oil [21]

Antibacterial and Antimicrobial properties of orange which fight bacteria-causing acne and avoiding future acne.

$\checkmark$ Orange has styptic properties which shrink and constrict excess oil and dirt from the pores.

$\checkmark$ Citric acid acts as a natural skin scrubber and is very effectual for depigmenting your skin.

$\checkmark$ When applied to the skin, it helps to boost healthy collagen production which causes firmer skin and reduces inflammation both of which cause a cascade of damage.

$\checkmark$ It fades post-breakout red marks by improving skin's natural healing response.

$\checkmark$ It increases the fruitfulness of your sunscreens and boosts your skin's defense against UV exposure.

$\checkmark$ It has alpha hydroxy acid (AHA), which exfoliates the skin and as a result of which it removes dead skin cells in the pores of the skin. 
ISSN No:-2456-2165

- Sugar

$\checkmark$ Sugar is a big depository of skin -beautifying qualities if it is used topically on the skin as an exfoliating scrub. It can deliver excellent results in brightening and helps in polishing your skin. It also has moisturizing properties that prevent the skin from drying. It also unclogs the clogged Pores.

\section{- Pistachio [22]}

Pistachios gain their reputation as free radical fighters and skincare and health product mainstays due to the extensive presence of phenolic antioxidant compounds. In addition, pistachios contain plant-based flavones that are another type of antioxidant which helps protect the body from damage done to skin cells by free radicals.

Free radicals are unstable atoms which are missing an electron, and often pair with other electrons. They are known to cause damage to the DNA within cells. When the damage is done to skin cells, there can be an impact on the cell's membrane and its mitochondria, which leads to the appearance of premature aging.

This "premature aging" is characterized by sagging skin, hyperpigmentation, age spots, loss of elasticity, fine lines and wrinkles, and other attributes which make a person look older than their actual chronological age.

This damage to the cell's genetic materials often includes affecting their growth, function, and ability to repair. The body has its own antioxidant defense against free radicals, but if the cells are overwhelmed by an abundance of free radicals, the body can't keep up.

Pistachio oil is influential antioxidant. It helps the body counteract free radicals to prevent key molecules in the skin cells from becoming damaged.

Pistachio Exfoliator is the fine powder of a pistachio shell. It is the simplest exfoliator because the shell is tough and when powdered, are often used as scrub into face wash, pack and lots of more.

- Benefits [23]

$\checkmark$ It removes dead skin cells and nourishes the skin deeply.

$\checkmark$ It removes blackheads and results in fresh and hydrated skin.

$\checkmark$ It can be used in lip scrub and body scrub as well.

\section{> 3D Modelling of Loofah Based Product}

For our proposed model of a loofah we have used the 3D CAD software Tinkercad. The design for our product is made up of primitive shapes that are either "solid" or "hole". Combining solids and holes together, required shapes has been created, which in turn leads to the proper design of the product.

We have taken the design of the product into careful consideration with a compartment for the shower gel, a plug/lid for refilling the shower gel, pores through which the gel can ooze out and bristles for the scrub.
With the use of such tools, the 3D model of the loofah based product has been possible.

The link to access the $3 \mathrm{D}$ model loofah is attached below.

https://www.tinkercad.com/things/2mplETb92vw

\section{RESULT}

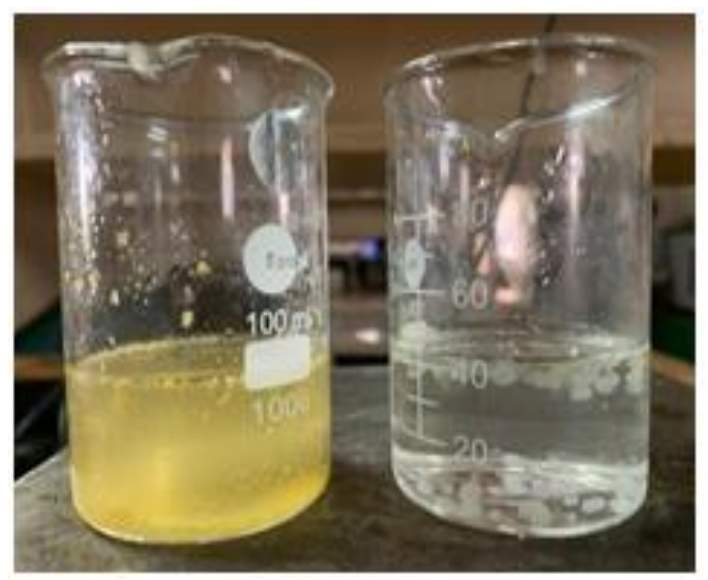

Fig 1:- Sodium alginate and calcium chloride solutions

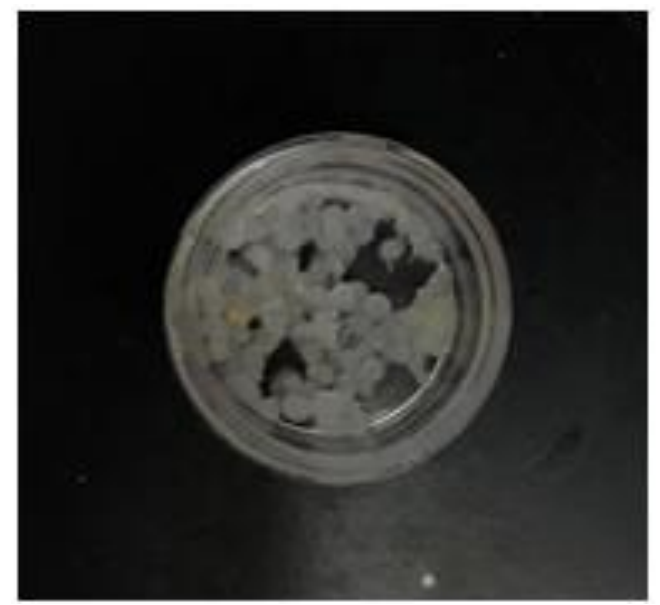

Fig 2:- Microbeads

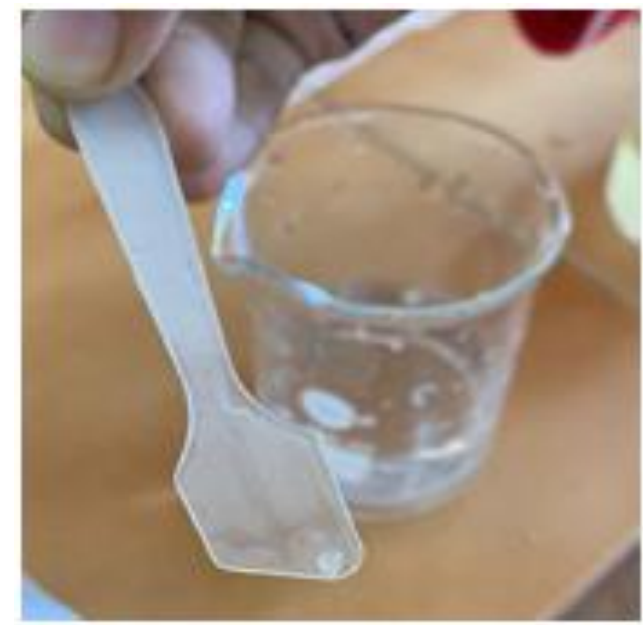

Fig 3:- Close up of the bead 
As shown in the pictures above we obtained the encapsulated orange peel powders and pistachio shell powder. These are very effectual and act as a good substituent to the micro-beads that are present in personal healthcare products and cosmetics. Also no irritant effects have been reported in humans due to sodium alginate. Alginates are high-molecular-weight polymers naturally occurring in brown algae. Even their use in feeding stuffs for fish is not expected to pose a risk for the environment. This helps in health making our product safer and heathier to use.

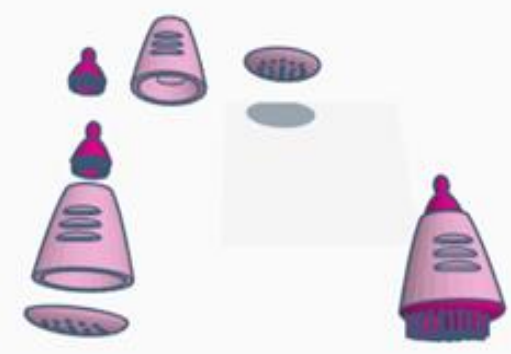

Fig 3:- 3D model of the loofah; the 3 parts and the overall design

The Loofah was designed to offer a simple hand-held loofah which makes using a loofah more convenient. The body of the loofah stores the shower gel and is made of rubber so that the shower gel can be squeezed out. The shower gel can be refillable into the compartment through a lid at one end of the loofah. The other end has a plastic lid with pores and the actual loofah bristles, through which the shower gel is squeezed into and lathers onto the loofah.

\section{CONCLUSION}

Plastic pollution in the marine ecosystem is an urgent and dreadful issue that needs to be addressed as it is found to be ubiquitous in the environment. Plastic has in literal terms invaded all ecological habitats and plastic waste is generated at an alarming rate. Due to its limitless applications and advantages they have found use in almost all fields like packaging, transport of gases and water, microbeads in cosmetics and insulation. But the dark side of plastic is inevitably its non-degradability. The harmful effects of plastic waste is ever growing, with the latest discovery being effect of micro plastics on marine aquatic life. There is ever increasing need to take strict measures at national and international levels in order to stop this menace, before it becomes too late. While countries like the USA have been the pioneers in banning microbeads, with several other countries following suit, several developing countries have not yet taken any steps towards its eradication. It therefore becomes essential for other solutions to be found, which include replacement of synthetic microbeads with natural alternatives such as the one proposed in this paper. This can encourage a nationwide ban on plastic microbeads.

\section{REFERENCES}

[1]. NEW EQUIPMENT AND NEW PRODUCTS: TREATMENT (INCLUDING DRUG AND SURGICAL EQUIPMENT AND SUPPLIES): American Journal of Physical Medicine: June 1980 https://journals.lww.com/ajpmr/Citation/1980/06000/ Shower_gel.34.aspx

[2]. How harmful is shower gel? Sugar and spice ; JAN 2017; https://www.sugarandspice.com.sg/post/howharmful-is-shower-gel

[3]. Rochman, C. M., Kross, S. M., Armstrong, J. B., Bogan, M. T., Darling, E. S., Green, S. J., ... Veríssimo, D. (2015). Scientific Evidence Supports a Ban on Microbeads. Environmental Science \& Technology, 49(18), 10759-10761. doi:10.1021/acs.est.5b03909

[4]. Leslie, H., 2014. Review of Microplastics in Cosmetics. Institute for Environmental Studies (IVM).

[5]. Baltic Marine Environment Protection Commission HELCOM. BASE ProjectImplementation of the Baltic Sea Action Plan in Russia, Technical Report Published for IVL Swedish Environmental Research Institute, August 2014; Swedish Environmental Research Institute: Stockholm, Sweden, 2014.

[6]. Magnusson, K.; Wahlberg, C. Screening of Microplastic Particles in and down-Stream of a Wastewater Treatment Plant, Technical Report published for IVL Swedish Environmental Research Institute, August 2014; Swedish Environmental Research Institute: Stockholm, Sweden, 2014.

[7]. Martin, C.; Eizhvertina, O. Quantitative Analysis of Microplastics in WWTP Effluent in the Niagara Region, Technical Report published for Niagara College Environmental TechnicianField and Lab (coop): Final Term Project, 2014; Niagara College Canada: Niagara-on-theLake, Canada, 2014.

[8]. Wardrop, P., Shimeta, J., Nugegoda, D., Morrison, P. D., Miranda, A., Tang, M., \& Clarke, B. O. (2016). Chemical Pollutants Sorbed to Ingested Microbeads from Personal Care Products Accumulate in Fish. Environmental Science \& Technology, 50(7), 40374044. doi:10.1021/acs.est.5b06280

[9]. Teuten, E. L.; Saquing, J. M.; Knappe, D. R.; Barlaz, M. A.; Jonsson, S.; Bjorn, A.; Rowland, S. J.; Thompson, R. C.; Galloway, T. S.; Yamashita, R.; Ochi, D.; Watanuki, Y.; Moore, C.; Viet, P. H.; Tana, T. S.; Prudente, M.; Boonyatumanond, R.; Zakaria, M. P.; Akkhavong, K.; Ogata, Y.; Hirai, H.; Iwasa, S.; Mizukawa, K.; Hagino, Y.; Imamura, A.; Saha, M.; Takada, H., Transport and release of chemicals from plastics to the environment and to wildlife. Philos Trans R Soc 2009, 364, (1526), 2027-45.

[10]. Ryan, P. G.; Connell, A. D.; Gardner, B. D., Plastic ingestion and PCBs in seabirds: Is there a relationship? Mar Poll Bull 1988, 19, (4), 174-176.

[11]. Yamashita, R.; Takada, H.; Fukuwaka, M.-a.; Watanuki, Y., Physical and chemical effects of ingested plastic debris on short-tailed shearwaters, Puffinus tenuirostris, in the North Pacific Ocean. Mar Poll Bull 2011, 62, (12), 2845-2849. 
[12]. Losada, S.; Roach, A.; Roosens, L.; Santos, F. J.; Galceran, M. T.; Vetter, W.; Neels, H.; Covaci, A., Biomagnification of anthropogenic and naturallyproduced organobrominated compounds in a marine food web from Sydney Harbour, Australia. Environ Int 2009, 35, (8), 1142-9.

[13]. Fendall, L.S., Sewell, M.A., 2009. Contributing to marine pollution by washing your face: microplastics in facial cleansers. Mar. Pollut. Bull. 58, 1225-1228.

[14]. Cheung, P. K., \& Fok, L. (2016). Evidence of microbeads from personal care product contaminating the sea. Marine Pollution Bulletin, 109(1), 582-585. doi:10.1016/j.marpolbul.2016.05.046 February 1994 - Mou-Tuan Huang, Chi-Tang Ho, Zhi Yuan Wang, Thomas Ferraro, You-Rong Lou, Kathe Stauber, Wei Ma, Constantino Georgiadis, Jeffrey D. Laskin and Allan H. Conney, Inhibition of Skin Tumorigenesis by Rosemary and Its Constituents Carnosol and Ursolic Acid---https://cancerres.aacrjournals.org/content/54/3/701.sh ort

[15]. W.S Goreja. SHEA BUTTER- The nourishing properties of Africa's best kept natural beauty secret--

https://books.google.co.in/books?hl=en\&lr=\&id=6iQj wr9v84gC\&oi=fnd\&pg=PA1\&dq=shea+butter + for + s kin\&ots $=$ AOHsHFCz0p\&sig $=89$ HE0SASUYiA_j9T1 bxqrAHb1_g\&redir_esc $=y \# v=$ onepage $\& q=$ shea $\% 20 \mathrm{~b}$ utter\%20for $\% 20$ skin $\& \mathrm{f}=$ false

[16]. March 2016 Microencapsulation of grape (Vitis labrusca var. Bordo) skin phenolic extract using gum Arabic, polydextrose, and partially hydrolyzed guar gum as encapsulating agent -- Luiza SiedeKuckCaciano Pelayo ZapataNore https://www.sciencedirect.com/science/article/pii/S03 08814615012649

[17]. December 14. 2018--- lifestyle magazine, Home » Lifestyle » Health \& Beauty » How to make your own eco-friendly and bio-active shower gel https://www.whatagreenlife.com/how-to-make-yourown-eco-friendly-and-bio-active-shower-gel/

[18]. 06 April 2020 .Cellulose acetate from oil palm empty fruit bunches waste as biodegradable microbeads for making scrubs ,Syarifah Nadrah Azzahra 1, b) and Dewi Tristantini ${ }^{1, a)}$ https://aip.scitation.org/doi/abs/10.1063/5.0005117

[19]. May 2017,Distribution and importance of microplastics in the marine environment: A review of the sources, fate, effects, and potential solutions ,H.S.Auta ${ }^{\mathrm{ab}}$ C.UEmenike ${ }^{\mathrm{bc}}$ S.HFauziah ${ }^{\mathrm{b}}$ https://www.sciencedirect.com/science/article/pii/S01 6041201631011X

[20]. Faith in Nature. The benefits of orange for our skin--https://www.faithinnature.co.uk/article/the-benefitsof-orange-for-skin.aspx

[21]. https://fashiongoalz.com/refreshing-orange-sugarscrub-for-dark-spots/

[22]. https://www.herbaldynamicsbeauty.com/blogs/herbaldynamics-beauty/pistachio-oil-benefits-for-skin-thewonders-of-vitamin-e
[23]. http://www.naturalexfoliator.com/Pistachio_Shell_Powder.html

[24]. Sharma, S., \& Chatterjee, S. (2017). Microplastic pollution, a threat to marine ecosystem and human health: a short review. Environmental Science and Pollution Research, 24(27), 21530 21547. doi:10.1007/s11356-017-9910-8 